\title{
Should I stay or should I go: honeybee drifting behaviour as a function of parasitism
}

\author{
Célia Bordier ${ }^{1}$, Maryline Pioz ${ }^{1,2}$, Didier Crauser ${ }^{1,2}$, Yves Le Conte ${ }^{1,2}$, \\ Cédric AlauX ${ }^{1,2}$ \\ ${ }^{1}$ INRA, UR406 Abeilles et Environnement, Domaine Saint-Paul, CS 40509, 84914, Avignon, France \\ ${ }^{2}$ UMT PrADE, CS 40509, 84914, Avignon, France
}

Received 2 May 2016 - Revised 4 August 2016 - Accepted 22 September 2016

\begin{abstract}
Nest drifting is often observed in honeybees (Apis mellifera) and can be detrimental to neighbouring colonies because it has the potential to increase disease transmission. However, the characteristics of drifting behaviour over a honeybee's lifetime and the influence of parasitism on this phenomenon have been insufficiently investigated. Using optical bee counters, we tracked the drifting behaviour of workers that were either infected with the parasite Nosema ceranae or uninfected. Approximately $10 \%$ of the tracked bees drifted into a foreign colony. The drifting prevalence was influenced by the colony's location in space but not by $N$. ceranae parasitism. However, the number and duration of drifts changed over the lifetime of the bees and the season, and parasitism had an effect on drifters, with Nosema -infected bees performing more but shorter drifts. This phenomenon was more pronounced in old bees ( +62 and $-15 \%$ for the number and duration of drifts, respectively) and could potentially be explained by the energetic stress induced by the parasite. In conclusion, combining a detailed analysis of drifting behaviour with the actual risk of newly established disease in colonies will benefit our knowledge of bee epidemiology.
\end{abstract}

\section{Apis mellifera / drifting / epidemiologic risk / Nosema ceranae / parasite}

\section{INTRODUCTION}

In social insects, individuals usually live in relatively closed societies to ensure that altruistic behaviours are directed towards related nestmates (Wilson 1971). However, drifting behaviour, described as the movement of individuals from their own nest to another, sometimes occurs either as a reproductive strategy or as an accidental phenomenon. On one hand, nest drifting in some eusocial insect species has been shown to represent an alternative reproductive strategy to maximise the

Electronic supplementary material The online version of this article (doi:10.1007/s13592-016-0475-1) contains supplementary material, which is available to authorized users.

Corresponding author: C. Bordier, celia.bordier@paca.inra.fr

Manuscript editor: Stan Schneider drifter's fitness either by contributing to brood raising in several related nests or by directly laying eggs in foreign nests (Neumann et al. 2003; LopezVaamonde et al. 2004; Nanork et al. 2005; Nanork et al. 2007; Sumner et al. 2007; Chapman et al. 2010; Takahashi et al. 2010; Blacher et al. 2013; O'Connor et al. 2013; Zanette et al. 2014). On the other hand, nest drifting can be an accidental phenomenon that is not deliberate but instead is the consequence of orientation errors during orientation or foraging flights, as frequently found in the honeybee, Apis mellifera (Free 1958; Pfeiffer and Crailsheim 1998; Neumann et al. 2000).

In the honeybee, nest drifting is believed to be involved in disease transmission between colonies (Neumann et al. 2000; Kralj and Fuchs 2006; Aubert et al. 2008; Kralj and Fuchs 2010). This phenomenon might increase disease transmission risk within apiaries, where the density is high compared to the density found in feral honeybee 
populations. The occurrence of drifting is often attributed to beekeeping practises (proximity and similarity of hives) as well as a lack of landmarks (Free 1958; Southwick and Buchmann 1995) and has been reported to vary depending on the season, hive characteristics and bee species (Duranville et al. 1991; Pfeiffer and Crailsheim 1998; Neumann et al. 2000; Paar et al. 2002; Neumann et al. 2003; Forfert et al. 2015). However, the role of parasites in drifting behaviour is not clear.

Because parasites can induce deficits in cognitive capacities and, as a consequence, affect homing ability (Gegear et al. 2006; Iqbal and Mueller 2007; Kralj et al. 2007), a higher occurrence of drifting is expected in infected bees. Nevertheless, Forfert et al. (2015) failed to show an association between the prevalence of drifting and parasitic infection by genotyping individuals from different colonies and determining their initial colony origin. However, deciphering the role of parasites in nest drifting, as well as the consecutive risk of disease transmission between neighbouring colonies, requires a detailed characterisation of this behaviour at the individual level. Notably, parasites can also lower the energetic state of individuals, leading to adverse effects on their flight activity (Kralj and Fuchs 2010; Alaux et al. 2014; Naug 2014; Wolf et al. 2014) and likely drifting behaviour.

The aim of the present study was therefore to provide a detailed analysis of drifting behaviour in honeybees over their lifetime and to test whether infection with a parasite could affect the rate, number and duration of nest drifts. We used a monitoring tool (optical bee counter) that was recently developed in our lab to continuously and automatically track the movements of individual bees from a colony to another colony over an extended period of time (Alaux et al. 2014). The influence of parasitism on drifting was determined by differentially infecting workers with the microsporidian Nosema ceranae. This parasite is transmitted by the oral route via trophallaxis or ingestion of contaminated comb material and food and develops in the epithelial cell layer of adult workers' midgut. $N$. ceranae can decrease the lifespan of bees (Fries 2010; Higes et al. 2010) and affect their flight activity: infected bees exhibit reduced orientation and homing ability and perform longer foraging trips (Kralj and Fuchs
2010; Alaux et al. 2014; Naug 2014; Wolf et al. 2014). This parasite was first described in the Asian honeybee Apis cerana and has been found in samples of the European honeybee Apis mellifera since the 1990s (Chen et al. 2008). Although it is not known when this shift in host species occurred, $N$. ceranae is now highly prevalent in European honeybee colonies and exhibits a worldwide distribution (Fries 2010; Higes et al. 2010), suggesting great potential for transmission and epidemiological risk between colonies.

\section{MATERIALS AND METHODS}

\subsection{Experimental setup}

Experiments were conducted from April to June 2014 at the Institut National de la Recherche Agronomique (INRA) in Avignon (France). We used colonies that were a mixture of the European subspecies typically used in the southeast of France for beekeeping, principally Apis mellifera mellifera .

We recorded the drifting behaviour of bees over their lifetime using three hives equipped with optical bee counters. The three hives were placed $1 \mathrm{~m}$ apart, and one empty hive was placed on either end as a landmark. The day before experimentation, frames with closed brood (at the last pupae stage) were removed from three other colonies, placed individually in boxes and kept in an incubator $\left(34{ }^{\circ} \mathrm{C}\right.$ and $70 \%$ humidity) overnight. The following day, newly emerged bees were collected and mixed to reduce potential colony effects. Half of the bees were artificially infected with $N$. ceranae to test the influence of parasitism on drifting behaviour (see below). Each bee was marked with a 3-mm wide tag number printed on laminated paper and glued (Sader $($ ) onto the thorax. The colour of the tags was specific to each colony (red, blue and green). For each colony and replicate, the drifting behaviour of 50 control bees not infected with Nosema and 50 Nosema -infected bees was recorded for 35 consecutive days. The study was performed simultaneously on the three hives and was repeated three times during the spring (in April, May and June) for a total of 450 control and 450 infected bees.

\subsection{Nosema infection}

Workers from Nosema -infected hives were collected to prepare a solution of fresh microsporidian spores. 
Spores were isolated as described by Higes et al. (2007). Briefly, pools of ten abdomens were crushed in distilled water, and the suspension was filtered and centrifuged to collect the spores. The spore concentration was determined using a haemocytometer chamber. Following the procedure described in Alaux et al. (2010), $N$. ceranae was identified by PCR analysis as the only species present in these bees.

Workers were individually infected on the day of their emergence by feeding with $2 \mu \mathrm{L}$ of $50 \%$ sucrose solution containing 100,000 Nosema spores, a dose currently found in naturally infected bees (Meana et al. 2010). To avoid the contamination of control bees, infected and control groups were placed separately in an incubator $\left(34{ }^{\circ} \mathrm{C}, 70 \%\right.$ relative humidity) for $5 \mathrm{~h}$, which is the time necessary to ingest the solution and to decrease the chance of having exchanges of crop content. The bees were subsequently introduced into the colonies.

To follow the Nosema proliferation, 50 additional emerging bees per treatment and colony were paintmarked on the thorax. They originated from the same colony sources than tracked bees, were introduced with tracked bees and were collected 9 days after the infection to determine Nosema spore levels. Generally, at this age, bees still perform in-hive tasks, and the infection is almost fully developed in the gut (Forsgren and Fries 2010).

\subsection{Optical bee counter}

To follow the individual flight activity of marked bees, we used the optical bee counters described in Alaux et al. (2014). Briefly, this monitoring technology is composed of a modified entrance with eight passages for bees, a camera at the entrance, a computer for image acquisition and software that processes the images and reports the in-and-out activity of bees at the hive entrance (patent number IDDN.FR.001.140013.000.R.P.2013.000.31235, INRA). For each detected bee, the software reports its ID (tag number and colour) and direction (whether it is going in or out of the hive), as well as the time of activity (day, hour, minute, and second). These data are converted into readable Excel files. Each detection is combined with a picture, which allows for verification of the data in case an identification failure is indicated in the Excel files.

\subsection{Drift characterisation}

In this study, a drift was recorded each time a bee entered a foreign hive, regardless of the time spent inside. Drifts from a foreign colony to another were also considered. Then, we determined the direction (origin and foreign colony), number and duration of drifts for each bee. The two colonies at the end of the row were defined as the edge colonies, and the central colony was defined as the inner colony. The influence of age on drifting behaviour was assayed by dividing the experimental period into three age groups: 1 to 10 days old (young bees), 11 to 20 days old (middle-age bees) and 21 to 35 days old (old bees). The impact of drifting on worker survival was also determined, and the rate of survival was calculated over 35 days using the day of the last record for a specific worker as the day of bee death.

\subsection{Data analysis}

All statistical analyses were performed using the statistical software R version 3.0.2 (R Development Core Team 2013). The effects of colony position, trial (April, May or June) and treatment on drifting behaviour were analysed using $\chi^{2}$ tests. Whether all drifting bees settled in the host colony was also assessed using $\chi^{2}$ tests. Nosema spore levels at 9 days and the impact of treatment on the age of first drift were assessed using Wilcoxon test.

Pseudo-replication occurred in our datasets because (i) the three colonies were sampled three times and (ii) a given bee was followed during her lifetime and was able to perform several drifts. Pseudo-replication can lead to erroneous conclusions, such as claiming that an effect of a treatment is real when it is not (Hurlbert 1984). To account for pseudo-replications in our experimental design, the influence of Nosema parasitism on drifting behaviour was analysed using mixed models. Models were compared using the small sample sizecorrected Akaike's information criterion (AICc) (Burnham and Anderson 2004). As recommended by Burnham and Anderson (2002), we considered that two nested models differing by less than 2 AICc points received identical support from the data. In such a situation, the model with fewer parameters was preferred. The probability of drifting for a bee (one drift or more vs no drift 
during its lifetime) was analysed by creating a general linear mixed model (glmer function of the lme4 $\mathrm{R}$ package) with a binomial distribution (Bates et al. 2013). Nosema infection, trial, and colony position were set as fixed explanatory variables, and colony number was set as the random explanatory variable. The coefficients of this model are the log of the odds ratios. Variations in the number and duration of drifts per age class were analysed using linear mixed models with a Gaussian error distribution (lmer function of the lmerTest package) (Kuznetsova et al. 2013). Nosema infection, age class, colony position and trial were analysed as fixed factors, whereas bee identity and colony were analysed as random factors. The mean, standard error and confidence interval of each covariable were calculated with the 'predict' function (type = 'response') for the first two and with the 'confint' function for the last.

Differences in survival probability between groups (drifting bees vs non-drifting bees and control vs Nosema ) were determined using Kaplan-Meier analysis ('survfit' function of the R survival package) (Therneau and Lumley 2014). Statistical significance was set at $P<0.05$.

\section{RESULTS}

Pathological analysis performed on 9-day-old bees showed that Nosema-infected bees had significantly more spores than control bees (control $520,000 \pm 799,249$ spores per bee; Nosema-infected bees: 7,575,556 \pm 4,631,973 spores per bee, Wilcoxon; $W=1, n=18, P<0.001)$.

\subsection{Drifting activity at the colony level}

Of the 900 tracked bees, we obtained information from 839 bees, and 61 bees were never recorded, likely due to early death or loss of tag. In total, flight activity for 422 control and 417 Nosema-infected bees was detected at least once. Of these bees, 49 control and 35 parasitized bees performed drifting behaviour (Table I). On average, $9.97 \pm 2.31 \%$ of bees drifted $(11.56 \pm 2.05 \%$ of control bees and $8.37 \pm 1.25 \%$ of Nosema infected bees). We recorded 1038 drifts, 545 $(52.5 \%)$ performed by control bees and 493 $(47.5 \%)$ by parasitized bees.

The position of a colony had a major influence on drifting behaviour. The percentage of drifting bees was higher in the inner colony than the edge colonies: 65.3 and $65.7 \%$ from the inner colony for control and parasitized bees, respectively (control: $\chi^{2}=23.31, P<0.001$; parasitized: $\chi^{2}=15.56$, $P<0.001)$. When considering the total number of drifts, the direction of drift was also highly biased, with 65.7 and $70.6 \%$ of drifts performed from the inner colony by the control and Nosema-infected bees, respectively (control: $\chi^{2}=282.63, P<0.001$; parasitized: $\left.\chi^{2}=329.90, P<0.001\right)$.

There was no effect of trial time on the percentage of drifting bees (Table I). The rate remained constant over the different trials (control: $\chi^{2}=1.15, P=0.56$, and parasitized: $\left.\chi^{2}=0.57, P=0.75\right)$. However, the number of drifts changed over the course of the experiment (control: $\chi^{2}=114.94, P<0.001$; parasitized: $\chi^{2}=6.93, P=0.03$ ). This change was especially pronounced for control bees, which

Table I. Drifting characteristics at the colony level and according to parasitism and colony.

\begin{tabular}{|c|c|c|c|c|c|c|}
\hline & \multicolumn{2}{|c|}{ Number of recorded bees } & \multicolumn{2}{|c|}{ Number and percentage of drifting bees } & \multicolumn{2}{|c|}{ Number of drifts } \\
\hline & Control & Nosema & Control & Nosema & Control & Nosema \\
\hline Colony 1 (edge) & 133 & 138 & $10(7.52 \%)$ & $6(4.35 \%)$ & 142 & 30 \\
\hline Colony 2 (inner) & 142 & 142 & $32(22.54 \%)$ & $23(16.20 \%)$ & 358 & 348 \\
\hline Colony 3 (edge) & 147 & 137 & $7(4.76 \%)$ & $6(4.38 \%)$ & 45 & 115 \\
\hline Trial 1 (April) & 145 & 143 & $20(13.79 \%)$ & $14(9.79 \%)$ & 233 & 191 \\
\hline Trial 2 (May) & 133 & 140 & $13(9.77 \%)$ & $11(7.86 \%)$ & 248 & 145 \\
\hline Trial 3 (June) & 144 & 134 & $16(11.11 \%)$ & $10(7.46 \%)$ & 64 & 157 \\
\hline
\end{tabular}


performed almost four times more drifts in April (trial 1) and May (trial 2) than in June (trial 3).

\subsection{Drifting activity at the individual level}

The percentage of drifting bees that settled in a foreign colony did not differ between the control (49\%) and Nosema-parasitized bees $(57.1 \%)$ $\left(\chi^{2}=0.27, P=0.61\right)$. Amongst the bees that did not settle in a foreign colony, the percentage of bees that came back to their initial colony or disappeared was similar between treatments (32.7 and $18.3 \%$, respectively, in the control groups, and 25.7 and $17.2 \%$ in Nosema groups; in both treatments: $\chi^{2}=0, P=1$ ).

\subsubsection{Drifting probability}

The probability of drifting was influenced by the colony position, but there was no effect of Nosema parasitism and trial (AICc model selection, Table SI). Bees from the inner colony tended to drift 3.7 times more often than bees from edge colonies (Table II).

Nosema parasitism did not influence the onset age for drifting (Wilcoxon; $W=774, n=84$, $P=0.45)$. Control and Nosema -parasitized bees

Table II. Estimated coefficients and $95 \%$ confidence interval (CI) of the selected models (see Table SI) investigating the influence of parasitism, age and colony position on drifting behaviour.

\begin{tabular}{|c|c|c|c|c|c|}
\hline Dependent variables & Covariate & Class & $\begin{array}{l}\text { Estimated } \\
\text { coefficient }\end{array}$ & $\begin{array}{l}\text { Lower } 95 \% \\
\text { CI }\end{array}$ & $\begin{array}{l}\text { Upper } 95 \% \\
\text { CI }\end{array}$ \\
\hline \multirow[t]{2}{*}{ Drifting probability } & Intercept & & -2.898 & -3.29 & -2.54 \\
\hline & Position & Inner & 1.472 & 0.97 & 1.99 \\
\hline \multirow[t]{11}{*}{ Drift numbers } & Intercept & & 4.659 & -1 & 10.33 \\
\hline & Age & 11-20 days & 3.028 & -4.23 & 10.34 \\
\hline & & 21-35 days & 8.526 & -2.95 & 19.90 \\
\hline & Treatment & Nosema & -3.739 & -10.1 & 2.57 \\
\hline & Position & Inner & -0.863 & -7.08 & 5.34 \\
\hline & Trial & May & 3.888 & -1.50 & 9.24 \\
\hline & & June & -1.977 & -7.38 & 3.39 \\
\hline & Age $\times$ Treatment & $11-20$ days $\times$ Nosema & 4.957 & -3.1 & 12.85 \\
\hline & & 21-35 days $\times$ Nosema & 7.404 & -4.25 & 19.21 \\
\hline & Age $\times$ Position & 11-20 days $\times$ Inner & 1.219 & -7.1 & 9.35 \\
\hline & & 21-35 days $\times$ Inner & -1.030 & -13.53 & 11.53 \\
\hline \multirow[t]{11}{*}{ Drift durations } & Intercept & & 3355.32 & NA & NA \\
\hline & Age & 11-20 days & 2253.68 & 121.8 & 5732.40 \\
\hline & & 21-35 days & -377.08 & -1455.48 & 3355.65 \\
\hline & Treatment & Nosema & 225.53 & -169.91 & 2560.96 \\
\hline & Position & Inner & 1039.20 & 639.25 & 3801.21 \\
\hline & Trial & May & 637.69 & -811.32 & 1651.15 \\
\hline & & June & -2885.26 & -4090.67 & -1957.29 \\
\hline & Age $\times$ Treatment & $11-20$ days $\times$ Nosema & -296.29 & NA & NA \\
\hline & & $21-35$ days $\times$ Nosema & -1555.16 & -4001.43 & 1178 \\
\hline & Age $\times$ Position & 11-20 days $\times$ Inner & -1473.26 & NA & NA \\
\hline & & 21-35 days $\times$ Inner & 355.08 & -2881.41 & 2843.34 \\
\hline
\end{tabular}


drifted for the first time when $10.1 \pm 5.5$ and $10.8 \pm 5.1$ days old, respectively.

\subsubsection{Drift number over bee lifetime}

The model with the lowest AICc was a model that underlined the significant effect of the trial and the age by treatment and age by colony position interactions on the number of drifts (Tables SI and II). The number of drifts per bee was maximum in May at 10.6 [9.53; 11.7] (mean predicted values with $95 \%$ confidence interval) and minimum in June at $6.1[5.2 ; 7]$. Overall, the number of drifts was slightly more important in Nosema -parasitized bees than in the control groups and increased with age: old bees exhibited more drifts than middle-age bees, which drifted more than young bees. This age- related increase was more important in parasitized bees, with old infected bees performing $62 \%$ more drifts than old control bees (Fig. 1).

\subsubsection{Drift duration over bee lifetime}

The model with the lowest AICc was the model that underlined the significant effect of the trial and the age by treatment and age by colony position interactions on the duration of drifts (Tables SI and II). The total drift duration per bee was higher in the second trial (May 5686 min $[5461 ; 5912]$ ) than in the first and third trials (April 4718 min [4378; 5050]; June $2034.9 \min$ [1780; 2289]). Overall, the time spent in a foreign colony was similar between parasitized and control bees (Fig. 2). However, Nosema infection caused a decrease in the drift

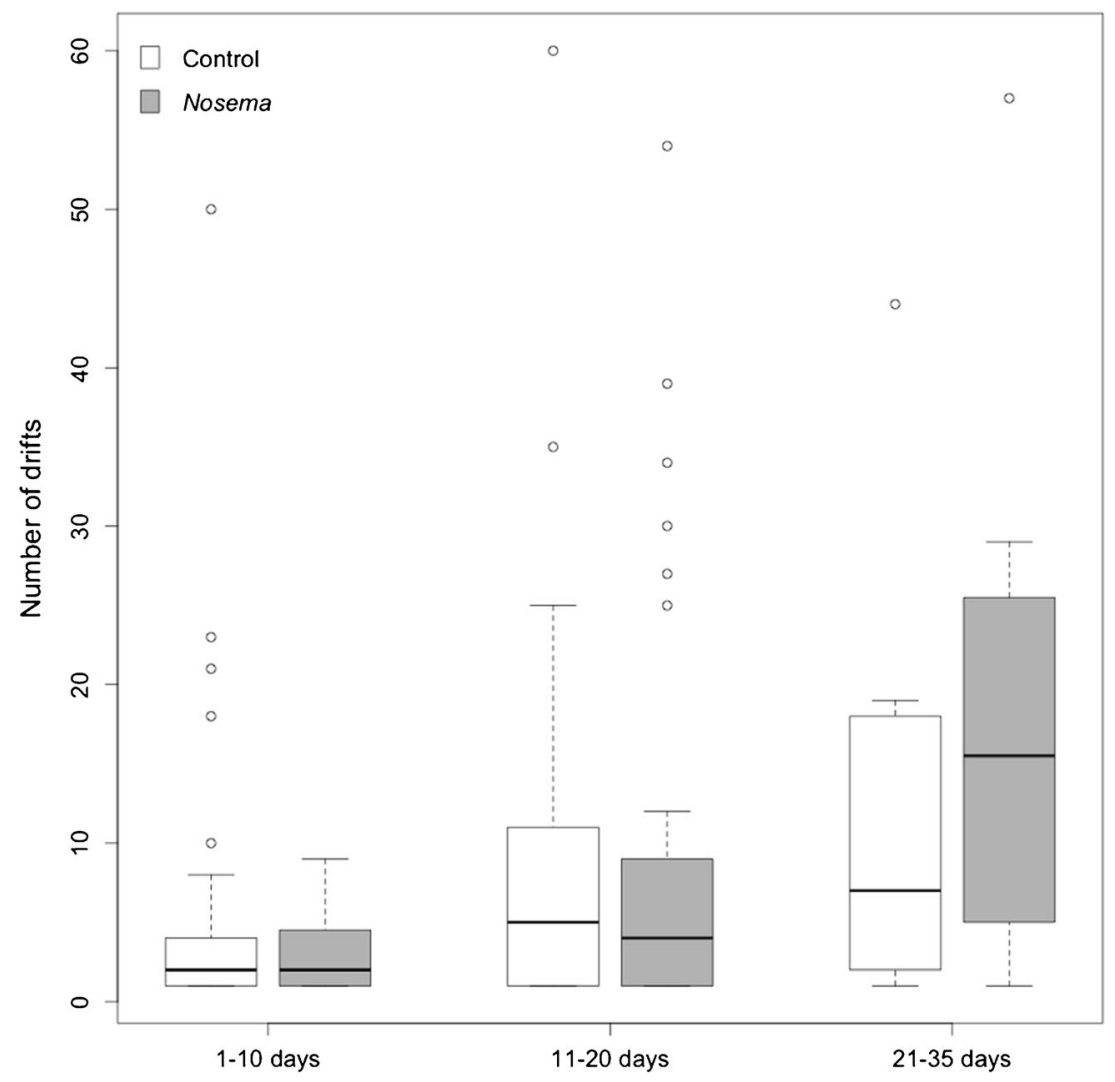

Figure 1. Cumulative number of drifts per bee as a function of age and treatment. The number of drifts cumulated within each age group ( 1 to 10 days old: $n=34$ and 20 for control and infected bees, respectively; 11 to 20 days old: $n=29$ and 28; 21 to 35 days old: $n=9$ and 8) is shown for all bees in control (non-parasitized) and Nosema parasitized drifting bees. Box plots show the first and third interquartile range with a line denoting the median. Whiskers encompass $90 \%$ of the individuals, beyond which outliers are represented by circles. 


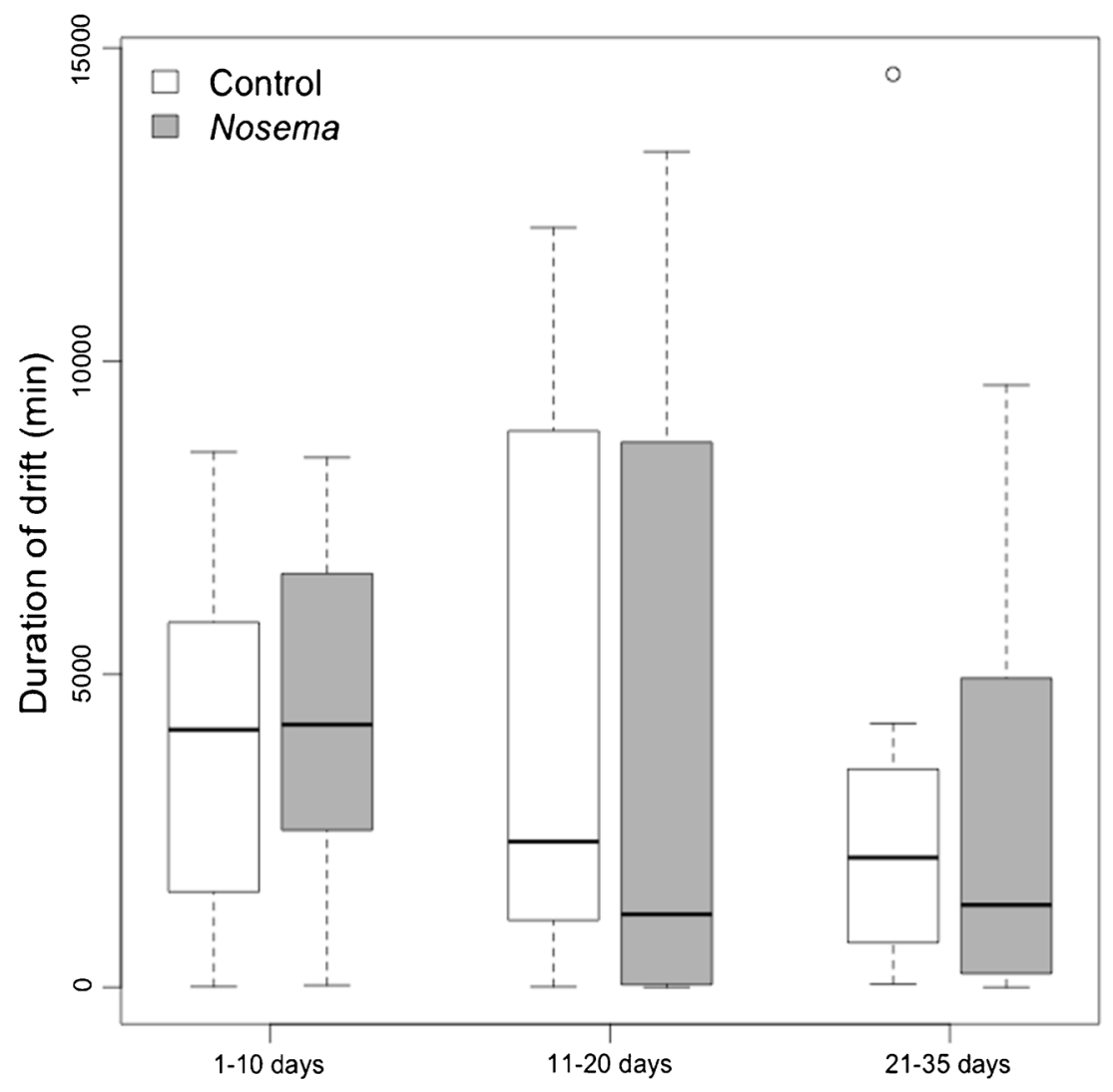

Figure 2. Cumulative drift duration per bee as a function of age and treatment. The duration of drifts cumulated within each age group ( 1 to 10 days old: $n=21$ and 13 for control and infected bees, respectively; 11 to 20 days old: $n=21$ and 19; 21 to 35 days old: $n=7$ and 7) is shown for all bees in control (non-parasitized) and Nosemaparasitized drifting bees. Box plots show the first and third interquartile range with a line denoting the median. Whiskers encompass $90 \%$ of the individuals, beyond which outliers are represented by circles.

duration in middle-age and old bees. For instance, in the latter age group, Nosema -parasitized bees spent $15 \%$ less time in a foreign colony during their drift than control bees.

\subsection{Survival}

As expected, the survival of Nosema-parasitized bees was significantly lower than that of control bees (Kaplan-Meier test, $P=0.002$ ). The survival rate of parasitized bees decreased faster than the control, especially after 15 days (Fig. 3).

We then tested whether drifting performance affected the survival probability of both control and parasitized bees. The survival probability of drifting and non-drifting bees was similar in both groups (Fig. 4a, b), and no effect of drifting on survival was detected (control: $P=0.62$; parasitized bees: $P=0.67$ ).

\section{DISCUSSION}

For the first time, by recording the in-and-out activity of bees between colonies, we were able to characterize the drifting behaviour of workers, including percentage, number and duration of drifts throughout their adult lifespan. We were able to test whether drifting behaviour is affected by Nosema parasitism. We found, like Forfert et al. (2015), that Nosema parasitism did not modify the probability of drifting. However, Nosema-parasitized drifters performed more but shorter drifts compared to 'healthy' drifters. 


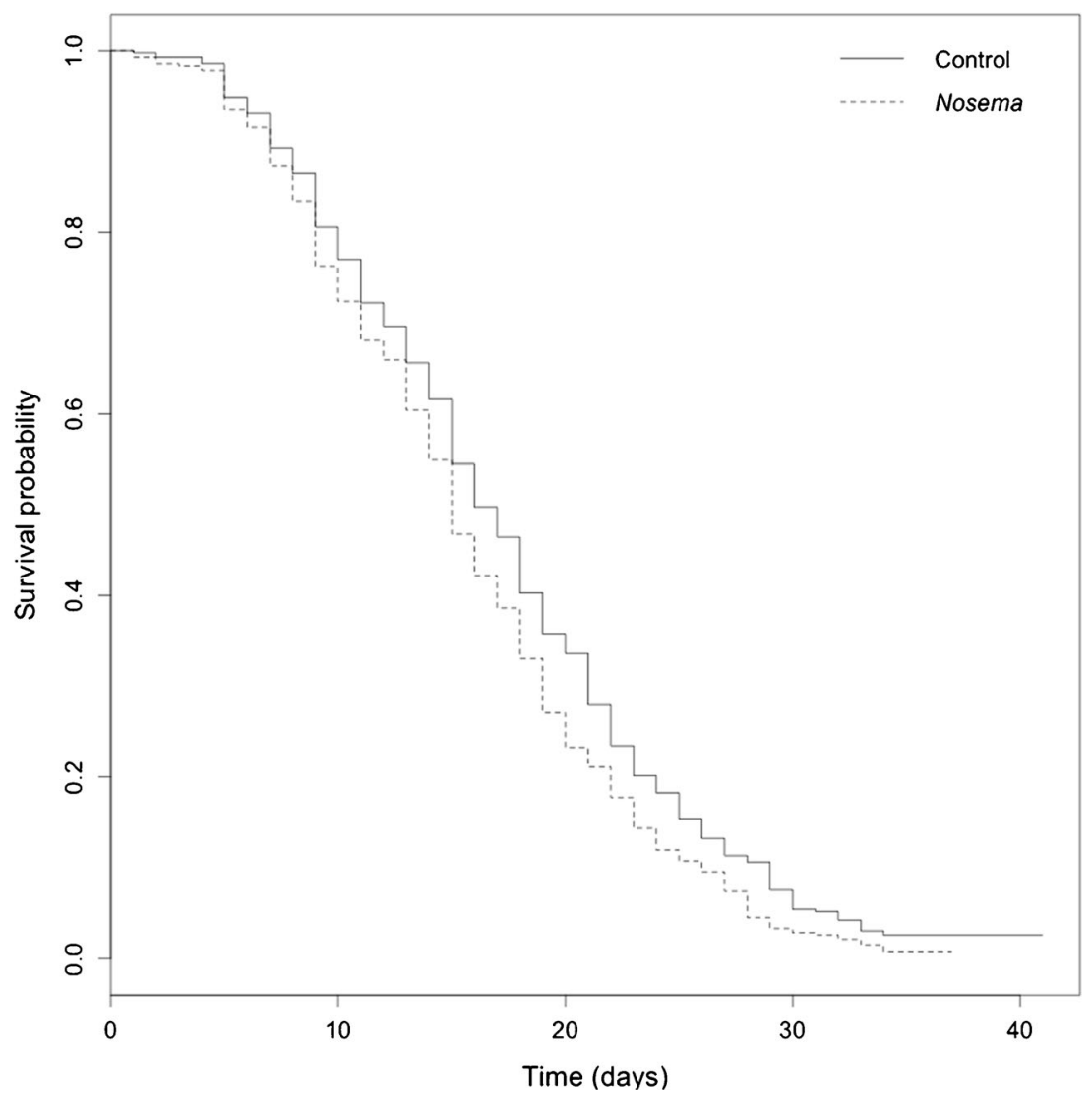

Figure 3. Effect of Nosema parasitism on bee survival. Data show the survival over 35 days for control and Nosema -parasitized bees (Kaplan-Meier test, $P=0.002$ ).

Until now, the study of drifting behaviour in honeybees was limited to punctual observations at

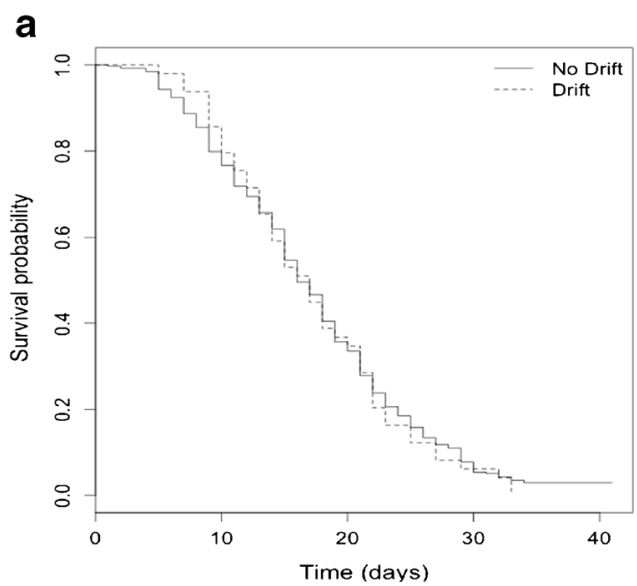

the colony level. The optical counter allowed us to follow the drifting dynamic between colonies

b

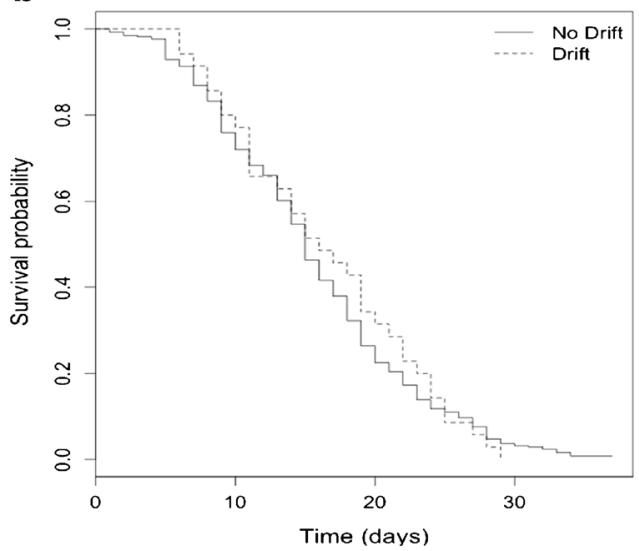

Figure 4. Effect of drifting performance on bee survival. Data show the survival of a non-drifting and drifting control bees (Kaplan-Meier test, $P=0.62$ ) and $\mathbf{b}$ non-drifting and drifting Nosema -parasitized bees (Kaplan-Meier test, $P=0.67)$. 
throughout the life of bees and to record this phenomenon at both the colony and individual levels. At the colony level, our results showed that $9.97 \pm 2.31 \%$ of a specific cohort drifted to a foreign colony. This percentage is within the range of previously published data (1 to $18 \%$ ) generated via individual genotyping or behavioural observations in European, Asian and African honeybees (Neumann et al. 2000; Paar et al. 2002; Neumann et al. 2003; Forfert et al. 2015). Colony position had a strong influence on drifting; bees drifted more from the inner colony to the edge colonies. This phenomenon is likely due to homogenous and similar landmarks around the inner colonies (Jay 1965; Pfeiffer and Crailsheim 1998; Forfert et al. 2015).

Drifting occurrence is generally attributed to orientation errors in honeybees, but the fact that 'only' $10 \%$ of bees drifted suggests that some bees are more prone to orientation errors than others and that this phenomenon does not occur randomly. For instance, it is well known that the tendency to perform some behavioural tasks in honeybees is influenced by their genetic and physiological backgrounds (Robinson and Huang 1998). This actually includes drifting since patrilines in honeybees can differ greatly in their tendency to drift (Duong and Schneider 2008). It is therefore possible that some patrilines exhibit poorer orientation abilities as compared to other ones. Determining the actual behaviour of drifting bees in their new host colony and their physiological background as done in bumblebees and wasps (Sumner et al. 2007; Blacher et al. 2013) would also help to better understand this drifting occurrence, e.g. do drifting bees still perform task activity or are inactive?

We found a seasonal effect on the drifting intensity at the colony level, with control bees performing more drifts during trials 1 and 2 (April-May) than in the trial 3 (June) (Table I). Drifting behaviour is a consequence of orientation errors and also depends on the acceptance level of the new host colony. Because the experimental design was the same between trials, changes in drifting intensity were likely the result of an increase in colony acceptance. In normal conditions, only $3.5 \%$ of incoming bees are checked by guard bees, but this permissiveness changes according to season, density of bees and nectar and pollen resources (Butler and Free 1952). For example, the acceptance levels of colonies increase when nectar flow is abundant but decrease during dearth periods (Downs and Ratnieks 2000). In this scenario, changes in drifting intensity should be associated with changes in resource abundance observed in the southeast of France between spring (blooming period, trials 1 and 2) and summer (dry period, trial 3).

At the individual level, we observed an agerelated increase in drift number and duration (time inside the foreign colony). The evolution of these parameters is in accordance with the age polyethism of bees (Seeley 1982), whereby young bees perform brood and nest care but switch to foraging activity (pollen, nectar and water collection) when they get older and thus are more likely to drift. We expected parasites to induce an increase in the propensity to drift, via either host manipulation (Adamo 2012; Biron \& Loxdale 2013) or deleterious effects on the host (e.g. impairment of cognitive functions). Indeed, it would be adaptive for $N$. ceranae to increase the bee propensity to drift and thus its transmission between colonies. Furthermore, the development of $N$. ceranae in bee guts affects the nutritional and energetic balance of bees (Mayack and Naug 2009; Aliferis et al. 2012; Dussaubat et al. 2012), which is expected to impair cognitive ability (Jaumann et al. 2013). However, behavioural (our study) and genetic analysis (Forfert et al. 2015) has demonstrated that drifting is not more prevalent in parasitized bees, although they can affect homing ability (Gegear et al. 2006; Iqbal and Mueller 2007; Kralj et al. 2007; Kralj and Fuchs 2010). A possible explanation is that the deficit in homing ability is not strong enough to increase orientation errors at the colony entrance. A non-mutually exclusive hypothesis is that parasitism reduces the homing success, as indicated by the higher mortality of infected bees, and therefore limits drifting prevalence.

Parasitism had an effect on drifters, with Nosema-parasitized bees performing more but shorter drifts. On one hand, the reduction of drift duration might reflect an increase in time spent in the field (Alaux et al. 2014; Naug 2014). The energy shortage induced by Nosema infection is suspected to decrease the flight ability of bees (Mayack and Naug 2010) and to result in prolonged resting periods (Wolf et al. 2014). The 
higher number of drifts could be explained by higher flight activity in Nosema-infected bees (Dussaubat et al. 2013) and the occurrence of orientation errors. Finally, the microsporidian parasite reduced, as expected, bee survival (Higes et al. 2007; Alaux et al. 2014), but drifting did not modify bee survival, as found by Pfeiffer and Crailsheim (1998) in one of their two trials. This result was confirmed in both parasitized and control bees, which suggests that drifting does not decrease the bee lifespan and guards have high tolerance for foreign bees, especially parasitized bees.

In conclusion, an average drifting rate of $10 \%$ within an apiary has the potential to influence the transmission of disease between colonies, especially if colonies do not discriminate infected individuals. However, the actual risk of disease transmission into foreign colonies by drifting bees remains to be investigated, as well as whether changes in drifting parameters caused by a parasite (e.g. more but shorter drifts) and seasonal variation can decrease or increase this transmission risk. These data will provide a better understanding of disease development and propagation between and within colonies.

\section{ACKNOWLEDGEMENTS}

We thank M. Coulon and F. Mondet for helpful comments on the manuscript. C. Bordier was supported by a Agence Nationale de la Recherche (ANR) project (ANR-13-ADAP-0002).

Author contributions C.B., C.A. and Y.L.C conceived the study, C.B., C.A. and D.C. conducted the experiments; C.B. and M.P. analysed the data; C.B. and C.A. wrote the manuscript.

Rester ou partir: comportement de dérive de l'abeille en réponse au parasitisme

Apis mellifera / dérive / risque épidémiologique / Nosema ceranae / parasite

Soll ich bleiben oder gehen: Verflug bei Honigbienen als Funktion von Parasitierung.

Apis mellifera / Verflug / epidemiologisches Risiko / Nosema ceranae / Parasiten

\section{REFERENCES}

Adamo, S.A., (2012) The strings of the puppet master: how parasites change host behavior., in: D. P. Hughes, J. Brodeur, F. Thomas (Eds), Host manipulation by parasites, Oxford University Press, UK, Chapter 3

Alaux, C., Brunet, J.L., Dussaubat, C., Mondet, F., Tchamitchan, S., Cousin, M., Brillard, J., Baldy, A., Belzunces, L.P., Le Conte, Y. (2010) Interactions between Nosema microspores and a neonicotinoid weaken honeybees (Apis mellifera) . Environ. Microbiol. 12, 774-782

Alaux, C., Crauser, D., Pioz, M., Saulnier, C., Le Conte, Y. (2014) Parasitic and immune-modulation of flight activity in honey bees tracked with optical counters. J. Exp. Biol. 217, 3416-3424

Aliferis, K.A., Copley, T., Jabaji, S. (2012) Gas chromatography-mass spectrometry metabolite profiling of worker honey bee (Apis mellifera L.) hemolymph for the study of Nosema ceranae infection. J. Insect Physiol. 58, 1349-1359

Aubert, M., Ball, B., Fries, I., Moritz, R., Milani, N., Bernardinelli, I. (2008). Virology and the honey bee. European Commission

Bates, D.M., Maechle, M., Bolker, B., Walker, S. (2013) Package lme4: Linear mixed-effects models using Eigen and S4. R package version 1.0-5

Biron, D.G., Loxdale, H.D. (2013) Host-parasite molecular cross-talk during the manipulative process of a host by its parasite. J. Exp. Biol. 216, 148-60

Blacher, P., Yagound, B., Lecoutey, E., Devienne, P., Chameron, S., Châline, N. (2013) Drifting behaviour as an alternative reproductive strategy for social insect workers. Proc. R. Soc. B Biol. Sci. 280, 20131888

Burnham, K.P., Anderson, D.R. (2002) Model selection and multi-model inference, a practical informationtheoretic approach. New York, NY: Springer- Verlag

Burnham, K.P., Anderson, D.R. (2004) Multimodel inference: understanding AIC and BIC in model selection. Sociol. Methods Res. 33, 261-304

Butler, C.G., Free, J.B. (1952) The behaviour of worker honeybees at the hive entrance. Behaviour 4, 262-292

Chapman, N.C., Beekman, M., Oldroyd, B.P. (2010) Worker reproductive parasitism and drift in the western honeybee Apis mellifera. Behav. Ecol. Sociobiol. 64, 419-427

Chen, Y., Evans, J.D., Smith, I.B., Pettis, J.S. (2008) Nosema ceranae is a long-present and wide-spread microsporidian infection of the European honey bee (Apis mellifera) in the United States. J. Invertebr. Pathol. 97, 186-188

Downs, S.G., Ratnieks, F. (2000) Adaptive shifts in honey bee (Apis mellifera L.) guarding behavior support predictions of the acceptance threshold model. Behav. Ecol. 11, 326-333

Duong, N., Schneider, S.S. (2008) Intra-patriline variability in the performance of the vibration signal and waggle dance in the honey bee, Apis mellifera. Ethology 114, 646-655. 
Duranville, C., Miniggio, C., Arnold, G., Borneck, R. (1991) Dérive des butineuses et réinfestation des colonies d'abeilles par Varroa jacobsoni. La santé l'abeille 125, 228-235

Dussaubat, C., Brunet, J.L., Higes, M., Colbourne, J.K., Lopez, J., et al. (2012) Gut pathology and responses to the microsporidium Nosema ceranae in the honey bee Apis mellifera. PLoS One 7, e37017

Dussaubat, C., Maisonnasse, A., Crauser, D., Beslay, D., Costagliola, G., Soubeyrand, S., Kretzchmar, A., Le Conte, Y. (2013) Flight behavior and pheromone changes associated to Nosema ceranae infection of honey bee workers (Apis mellifera) in field conditions. J. Invertebr. Pathol. 113, 42-51

Forfert, N., Natsopoulou, M.E., Frey, E., Rosenkranz, P., Paxton, R.J., Moritz, R.F.A. (2015) Parasites and pathogens of the honeybee (Apis mellifera) and their influence on inter-colonial transmission. PLoS One 10, e0140337

Forsgren, E., Fries, I. (2010) Comparative virulence of Nosema ceranae and Nosema apis in individual European honey bees. Vet. Parasitol. 170, 212-217

Free, J.B. (1958) The drifting on honey-bees. J. Agric. Sci. 51, 294-306

Fries, I. (2010) Nosema ceranae in European honey bees (Apis mellifera) . J. Invertebr. Pathol. 103, 73-79

Gegear, R.J., Otterstatter, M.C., Thomson, J.D. (2006) Bumble-bee foragers infected by a gut parasite have an impaired ability to utilize floral information. Proc. Biol. Sci. 273, 1073-1078

Higes, M., García-Palencia, P., Martín-Hernández, R., Meana, A. (2007) Experimental infection of Apis mellifera honeybees with Nosema ceranae (Microsporidia). J. Invertebr. Pathol. 94, 211-217

Higes, M., Martín-Hernández, R., Meana, A. (2010) Nosema ceranae in Europe: an emergent type C nosemosis. Apidologie 41, 375-392

Hurlbert, S.H. (1984) Pseudoreplication and the design of ecological field experiments. Ecol. Monogr. 54, 187211

Iqbal, J., Mueller, U. (2007) Virus infection causes specific learning deficits in honeybee foragers. Proc. Biol. Sci. $274,1517-1521$

Jaumann, S., Scudelari, R., Naug, D. (2013) Energetic cost of learning and memory can cause cognitive impairment in honeybees. Biol. Lett. 9, 20130149

Jay, S.C. (1965). Drifting of honeybees in commercial apiaries. I. Effect of various environmental factor. J. Apic. Res. 4, 167-175

Kralj, J., Fuchs, S. (2006) Parasitic Varroa destructor mites influence flight duration and homing ability of infested Apis mellifera foragers. Apidologie 37, 577-587

Kralj, J., Fuchs, S. (2010) Nosema sp. influences flight behavior of infected honey bee (Apis mellifera) foragers. Apidologie 41, 21-28

Kralj, J., Brockmann, A., Fuchs, S., Tautz, J. (2007) The parasitic mite Varroa destructor affects non-associative learning in honey bee foragers, Apis mellifera L. J.
Comp. Physiol. A Neuroethol. Sensory, Neural, Behav. Physiol. 193, 363-370

Kuznetsova, A., Brockhoff, P.B., Bojesen Christensen, R.H. (2013) Package "ImerTest". Tests for random and fixed effects for linear mixed effect models (lmer objects of lme4 package). R package version 2.0-3

Lopez-Vaamonde, C., Koning, J.W., Brown, R.M., Jordan, W.C., Bourke, A.F.G. (2004) Social parasitism by male-producing reproductive workers in a eusocial insect. Nature 430, 557-560

Mayack, C., Naug, D. (2009) Energetic stress in the honeybee Apis mellifera from Nosema ceranae infection. J. Invertebr. Pathol. 100, 185-188

Mayack, C., Naug, D. (2010) Parasitic infection leads to decline in hemolymph sugar levels in honeybee foragers. J. Insect Physiol. 56, 1572-1575

Meana, A.M., Martín-Hernández, R., Higes, M. (2010) The reliability of spore counts to diagnose Nosema ceranae infections in honey bees. J. Apic. Res. 49, 212-214

Nanork, P., Paar, J., Chapman, N.C., Wongsiri, S., Oldroyd, B.P. (2005) Entomology: Asian honeybees parasitize the future dead. Nature 437, 829

Nanork, P., Chapman, N.C., Wongsiri, S., Lim, J., Gloag, R.S., Oldroyd, B.P. (2007) Social parasitism by workers in queenless and queenright Apis cerana colonies. Mol. Ecol. 16, 1107-1114

Naug, D. (2014) Infected honeybee foragers incur a higher loss in efficiency than in the rate of energetic gain. Biol. Lett. 10, 20140731

Neumann, P., Moritz, R.F., Mautz, D. (2000) Colony evaluation is not affected by drifting of drone and worker honeybees (Apis mellifera L.) at a performance testing apiary. Apidologie 31, 67-79

Neumann, P., Radloff, S.E., Pirk, C.W.W., Hepburn, R. (2003) The behaviour of drifted Cape honeybee workers (Apis mellifera capensis): predisposition for social parasitism? Apidologie 34, 585-590

O’Connor, S., Park, K.J., Goulson, D. (2013) Worker drift and egg dumping by queens in wild Bombus terrestris colonies. Behav. Ecol. Sociobiol. 67, 621-627

Paar, J., Oldroyd, B.P., Huettinger, E., Kastberger, G. (2002) Drifting of workers in nest aggregations of the giant honeybee Apis dorsata. Apidologie 33, 553-561

Pfeiffer, K.J., Crailsheim, K. (1998) Drifting of honeybees. Insectes Soc. 45, 151-167

R Development Core Team. (2013) R: a language and environment for statistical computing. R Foundation for Statistical Computing, Vienna, Austria

Robinson, G.E., Huang, Z.Y. (1998) Colony integration in honey bees: genetic, endocrine and social control of division of labor. Apidologie 29, 159-170

Seeley, T.D. (1982) Adaptive significance of the age polyethism schedule in honeybee colonies. Behav. Ecol. Sociobiol. 11, 287-293

Southwick, E.E., Buchmann, S.L. (1995) Effects of horizon landmarks on homing success in honey bees. Am. Nat. 146, 748 
Sumner, S., Lucas, E., Barker, J., Isaac, N. (2007) Radio-tagging technology reveals extreme nestdrifting behavior in a eusocial insect. Curr. Biol. 17, 140-145

Takahashi, J.I., Martin, S.J., Ono, M., Shimizu, I. (2010) Male production by non-natal workers in the bumblebee, Bombus deuteronymus (Hymenoptera: Apidae). J. Ethol. 28, 61-66

Therneau, T.M., Lumley, T. (2014) Package "survival". Survival analysis. R package version 2.37-7
Wilson, E.O. (1971) The insect societies. Cambridge, Massachusett: Harvard University Press

Wolf, S., McMahon, D.P., Lim, K.S., Pull, C.D., Clark, S.J., Paxton, R.J., Osborne, J.L. (2014) So near and yet so far: harmonic radar reveals reduced homing ability of nosema infected honeybees. PLoS One 9, e103989

Zanette, L.R.S., Miller, S.D.L., Faria, C.M.A., LopezVaamonde, C., Bourke, A.F.G. (2014) Bumble bee workers drift to conspecific nests at field scales. Ecol. Entomol. 39, 347-354 